\title{
Tracking of Virus Particles in Time-Lapse Fluorescence Microscopy Image Sequences
}

\author{
W. J. Godinez ${ }^{1}$, M. Lampe ${ }^{2}$, S. Wörz ${ }^{1}$, B. Müller ${ }^{2}$, R. Eils ${ }^{1}$ and K. Rohr ${ }^{1}$ \\ ${ }^{1}$ University of Heidelberg, IPMB, and DKFZ Heidelberg, Dept. Bioinformatics and \\ Functional Genomics, 69120 Heidelberg, Germany \\ ${ }^{2}$ University of Heidelberg, Dept. of Virology, 69120 Heidelberg, Germany \\ Email: wgodinez@ieee.org
}

\begin{abstract}
Modern developments in time-lapse microscopy enable the observation of a variety of processes exhibited by viruses. The dynamic nature of these processes requires the tracking of viruses over time to explore the spatio-temporal relationships. In this work, we developed deterministic and probabilistic approaches for multiple virus tracking. A quantitative comparison based on synthetic image sequences was carried out to evaluate the performance of the different algorithms. We have also applied the algorithms to real microscopy images of HIV-1 particles and have compared the tracking results with ground truth obtained from manual tracking. It turns out that the probabilistic approach outperforms the deterministic schemes.
\end{abstract}

\section{Introduction}

Exploration of the spatio-temporal relationships of viruses advances the understanding of viral infections, e.g., HIV-1. Modern time-lapse microscopy of these processes results in a large amount of visual data that, on the one hand, provides the basis for a solid statistical analysis, yet, on the other hand, requires automatic image analysis methods. However, the task of virus tracking is hampered by various issues. For instance, viruses are relatively small and exhibit a complex motion behavior, in particular, abrupt changes in velocity and direction are observable; this precludes the usage of motion constraints often employed in other tracking applications. Another problem is the relatively large number of viruses in the image sequences, which rules out the usage of algorithms that are only applicable in the case of one or few objects. A further problem is the low signal-to-noise ratio (SNR), which hinders the accurate localization of particles.

Only few approaches for virus tracking have been described in the literature. Typically, a deterministic two-step approach is used consisting of: 1) virus detection, and 2) correspondence finding. For virus detection, most of the algorithms employ some kind of maximum intensity search strategy, in which the position of viruses are associated with intensity peaks. Subsequently, thresholding techniques or techniques based on the intensity moments of detected candidate viruses (e.g., [1]) are employed for rejecting noise-induced maxima. The position of the particles may be refined by model fitting (e.g., [2]). For correspondence 
finding, a nearest-neighbor model, which assumes that a single virus carries out the smallest possible displacement between two consecutive time steps, is typically employed. However, in image regions with a high density of viruses, the search for correspondences becomes ambiguous, since several possibilities are plausible. To address this issue, approaches that consider the motion of all viruses between two consecutive time frames have been introduced. For instance, in [1] the total distance between all viruses is minimized.

We introduce deterministic and probabilistic algorithms for tracking multiple viruses in microscopy time-lapse images. For the first time, a particle filter approach has been employed for the task of virus tracking.

\section{Tracking of multiple virus particles}

We have developed four two-step deterministic tracking approaches for virus particle tracking by combining two virus detection schemes and two correspondence finding techniques. The first virus detection scheme is comprised by the application of the spot-enhancing filter ("SEF") [3], based on the Laplacian-ofGaussian filter, and a thresholding step, which yields a binarized image from which viruses can be detected. The second technique is an enhanced 2D Gaussian fitting algorithm ("Gauss") along with rejection criteria for noise-induced candidate viruses, such as minimum integrated intensity, and maximum ellipticity of the fitted 2D Gaussian function. For correspondence finding, we have investigated an algorithm based on a smooth motion model along with a greedy optimization step ("Smooth") [4], and an algorithm based on a nearest-neighbor scheme with an optimization step inspired by an algorithm for the transportation problem ("NNeigh") [1]. The first algorithm ("Smooth") assumes a gradual change in direction and displacement, and only considers the motion of one virus at a time to resolve correspondence conflicts. The second algorithm ("NNeigh") assumes small displacements between two consecutive time-frames, and minimizes the overall frame-to-frame displacement induced by all viruses.

We have also developed a probabilistic approach for multiple virus tracking, which formulates the task of tracking as a Bayesian sequential estimation problem. Such probabilistic approaches are relatively novel in the area of biological imaging. An initial effort in this direction is introduced in [5], in which a joint particle filter is utilized for a different tracking application, namely the tracking of growing microtubules. We have extended and applied this method for the tracking of multiple viruses. In our case, each virus is represented by a state vector $\mathbf{x}_{t}$ that includes the position, velocity, and intensity. This state evolves in time according to a predefined dynamic model $p\left(\mathbf{x}_{t} \mid \mathbf{x}_{t-1}\right)$ (here we used Brownian dynamics) and is observed at discrete times $t$ through measurement vectors $\mathbf{y}_{t}$. The predictions generated by the dynamic model are tested against the information provided by the measurement $\mathbf{y}_{t}$ via an observation model $p\left(\mathbf{y}_{t} \mid \mathbf{x}_{t}\right)$ (in our case, the probability that the predicted state $\mathbf{x}_{t}$ generated a Gaussian intensity distribution in the image, i.e., a virus). Inference on the true state is based on the posterior distribution $p\left(\mathbf{x}_{t} \mid \mathbf{y}_{1: t}\right)$, which is conditioned on a sequence of 
Fig. 1. Tracking results for a synthetic image sequence

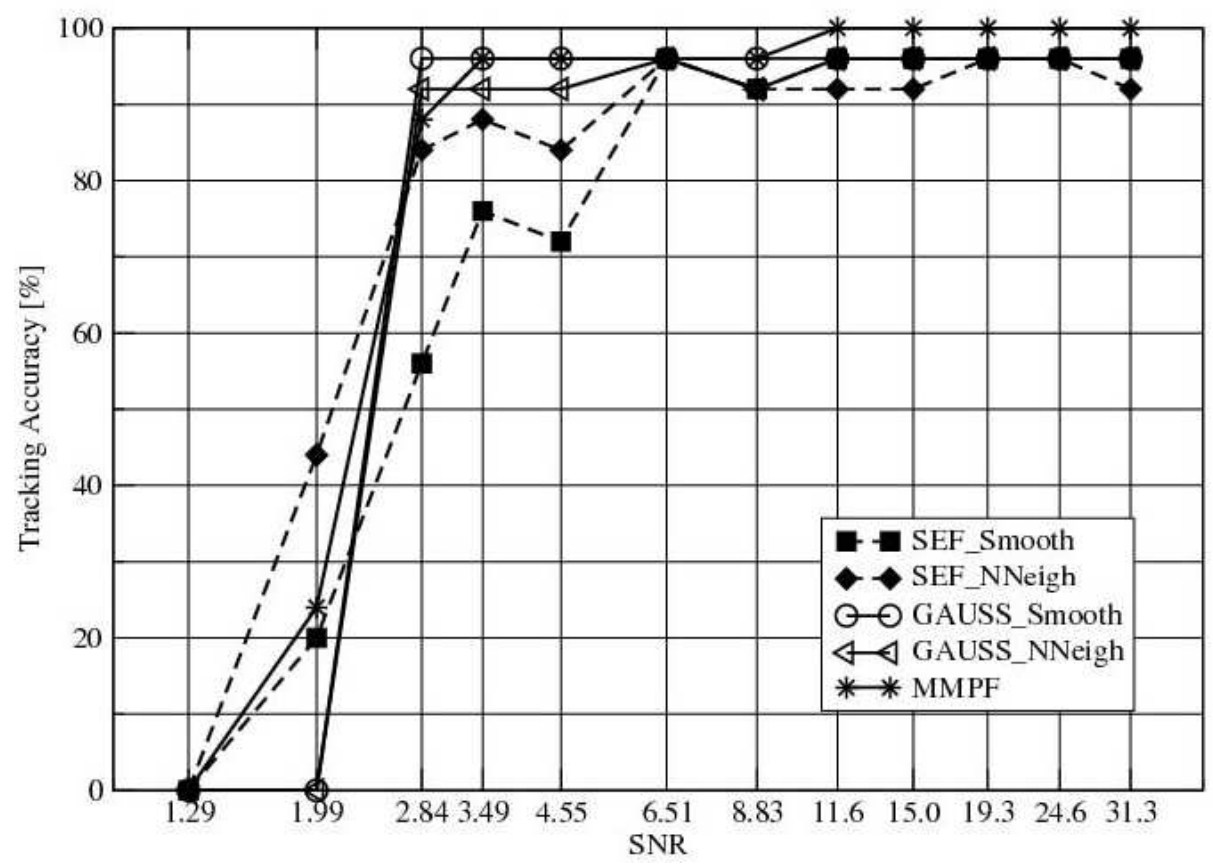

observations $\mathbf{y}_{1: t-1}$, and which is obtained using Bayes' theorem:

$$
\begin{gathered}
p\left(\mathbf{x}_{t} \mid \mathbf{y}_{1: t-1}\right)=\int p\left(\mathbf{x}_{t} \mid \mathbf{x}_{t-1}\right) p\left(\mathbf{x}_{t-1} \mid \mathbf{y}_{1: t-1}\right) d \mathbf{x}_{t-1} \\
p\left(\mathbf{x}_{t} \mid \mathbf{y}_{1: t}\right) \propto p\left(\mathbf{y}_{t} \mid \mathbf{x}_{t}\right) p\left(\mathbf{x}_{t} \mid \mathbf{y}_{1: t-1}\right)
\end{gathered}
$$

The numeric implementation of these equations has been done via a joint particle filter. In contrast to [5], in which manual initialization is employed, in our algorithm we automatically initialize the tracking via one of the above-mentioned virus detection approaches. Furthermore, instead of using a fixed value for the $\sigma$ parameter which defines the Gaussian intensity distribution in the observation model $p\left(\mathbf{y}_{t} \mid \mathbf{x}_{t}\right)$, we automatically compute this parameter based on the Gaussian fitting results; in this way, we can track virus particles with different apparent sizes.

\section{Experimental results}

We have applied our algorithms to both synthetic as well as real microscopy image sequences. In total, we generated four synthetic image sequences, each consisting of 50 images (size $400 \times 400$ pixels) containing 25-40 simulated moving viruses using 12 different signal-to-noise ratio (SNR) levels. The noise model was assumed to be Poisson distributed. To measure the performance, we employed 
Table 1. Tracking results for a real microscopy image sequence

\begin{tabular}{cccccc}
\hline & SEF+Smooth & SEF+NNeigh & Gaussian+Smooth & Gaussian+NNeigh & MMPF \\
\hline$P_{\text {track }}$ & $60.87 \%$ & $73.91 \%$ & $60.87 \%$ & $69.57 \%$ & $95.65 \%$ \\
\hline
\end{tabular}

Fig. 2. Sample image from a real microscopy image sequence (a) and results obtained by the MMPF (b). An enlarged section delineated with a black rectangle in (b) is shown in (c). The image intensities have been inverted for visualization purposes

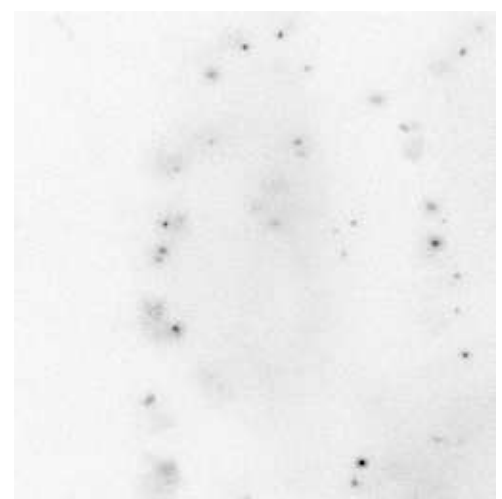

(a) $\mathrm{t}=0$

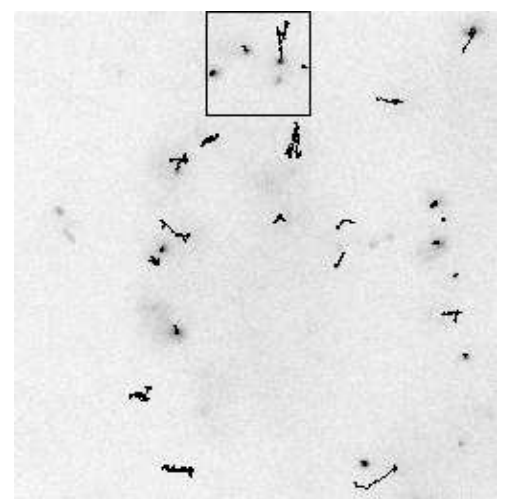

(b) $\mathrm{t}=138$

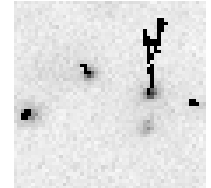

(c) $t=138$

the tracking accuracy defined as $P_{\text {track }}=\frac{n_{\text {track,correct }}}{n_{\text {track,total }}}$, which reflects the ratio between the number of completely correctly computed trajectories $n_{\text {track,correct }}$ and the number of all true trajectories $n_{\text {track,total }} \cdot n_{\text {track,correct }}$ counts those trajectories that span the same time period as their true counterparts, and yield a mean-squared error (MSE) between the measured object displacement and the true object displacement lower than a certain threshold (in our case we used a threshold of 2). A statistical test (one-way ANOVA) for each of the synthetic sequences yielded no significant differences between the performance of the different algorithms (see Fig. 1). The reason for this result is probably the simplistic nature of the synthetic images (e.g., constant number of objects and constant intensity). We also validated the algorithms based on a real microscopy image sequence (see Fig. 2), consisting of 250 16-bit images (size $512 \times 512$ pixels) with 23 well-defined virus particles. In this sequence, fluorescently labeled HIV-1 particles [6] were imaged using a fluorescence widefield microscope; fluorophores were excited with their respective excitation wavelengths and movies were recorded with a frequency of $10 \mathrm{~Hz}$. Ground truth on the virus positions was obtained by manual tracking using the commercial software MetaMorph. The experimental results, which have been listed in Table 1, indicate that the probabilistic approach $(\mathrm{MMPF}=$ multimodal particle filter $)$ outperforms the deterministic approaches (95.65\% accuracy vs. $73.91 \%$ and $69.57 \%$ for the two best deterministic approaches, Fig. 2). 


\section{Discussion}

We have developed deterministic and probabilistic approaches for the tracking of viruses in microscopy images sequences. Our quantitative comparison based on real microscopy image sequences shows that among all four deterministic approaches, the combinations of spot-enhancing filter (SEF) or Gaussian fitting with an enhanced nearest-neighbor motion model achieve the best results. Overall, the probabilistic approach based on a particle filter outperforms the deterministic schemes. The developed algorithms provide information on the displacements, sizes, and intensities of the virus particles. Nevertheless, there are still some open issues. For instance, in areas of high object density with fast changing dynamics all algorithms have difficulties in determining the correct correspondences. In future work, the performance of the algorithms should be further improved in this regard.

\section{References}

1. Sbalzarini IF, Koumoutsakos P. Feature point tracking and trajectory analysis for video imaging in cell biology. J Struct Biol 2005;151(2):182-195.

2. Seisenberger G, Ried MU, Endre T, Buning H, Hallek M, Bräuchle C. Real-time single-molecule imaging of the infection pathway of an adeno-associated virus. Science 2001;294(5548):1929-1932.

3. Sage D, Neumann FR, Hediger F, Gasser SM, Unser M. Automatic tracking of individual fluorescence particles: application to the study of chromosome dynamics. IEEE Trans Image Process 2005;14(9):1372-1382.

4. Chetverikov D, Verestoy J. Tracking feature points: a new algorithm. Int Conf Pattern Recognit 1998;02:1436.

5. Smal I, Niessen W, Meijering E. Bayesian tracking for fluorescence microscopic imaging. Procs ISBI 2006; 550-553.

6. Lampe M, Briggs JAG, Endress T, Glass B, Riegelsberger S, Kr" ausslich H, et al. Double-labelled HIV-1 particles for study of virus-cell interaction. Virology 2006;In Press. 